\title{
Pressure tensor of a spherical interface
}

Cite as: J. Chem. Phys. 97, 3576 (1992); https://doi.org/10.1063/1.462992

Submitted: 21 February 1992 . Accepted: 12 May 1992 . Published Online: 31 August 1998

Edgar M. Blokhuis, and Dick Bedeaux

\section{ARTICLES YOU MAY BE INTERESTED IN}

The Statistical Mechanical Theory of Surface Tension

The Journal of Chemical Physics 17, 338 (1949); https://doi.org/10.1063/1.1747248

The Statistical Mechanical Theory of Transport Processes. IV. The Equations of Hydrodynamics

The Journal of Chemical Physics 18, 817 (1950); https://doi.org/10.1063/1.1747782

A molecular dynamics study of liquid drops

The Journal of Chemical Physics 81, 530 (1984); https://doi.org/10.1063/1.447358

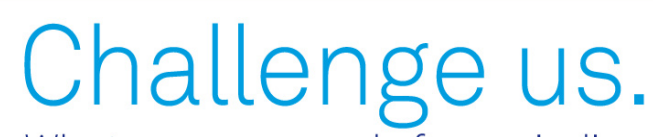

What are your needs for periodic signal detection?
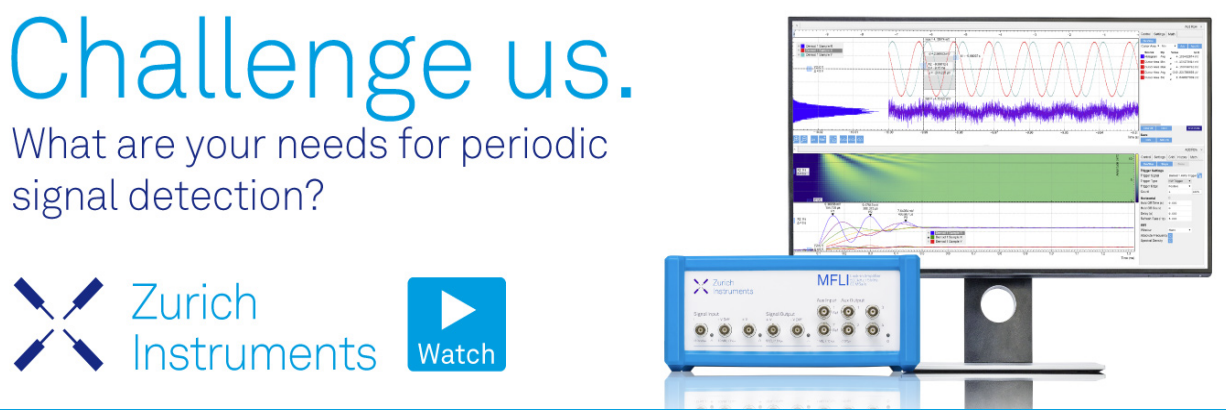

J. Chem. Phys. 97, 3576 (1992); https://doi.org/10.1063/1.462992 


\title{
Pressure tensor of a spherical interface
}

\author{
Edgar M. Blokhuis and Dick Bedeaux \\ Department of Physical and Macromolecular Chemistry, Gorlaeus Laboratories, University of Leiden, 2300 \\ RA Leiden, The Netherlands
}

(Received 21 February 1992; accepted 12 May 1992)

\begin{abstract}
In this paper we show how the use of the Irving-Kirkwood expression for the pressure tensor leads to expressions for the pressure difference, the surface tension of the flat interface, and the Tolman length which agree with the expressions found using microscopic sum rules. The use of the Schofield-Henderson expression for the pressure tensor for a particular contour different from the contour that leads to the Irving-Kirkwood expression is found to give incorrect results for the pressure difference and, in particular, also for the Tolman length. The distance between the so-called mechanical surface of tension and the Gibbs dividing surface is found not to be given by Tolman's length. Using an approximate expression for the pair density it is possible to find values for the location of the mechanical surface of tension and for Tolman's length which are in reasonably good agreement with values found by Nijmeijer et al. in molecular dynamics simulations.
\end{abstract}

\section{INTRODUCTION}

The description of the influence of curvature on the thermodynamic properties of interfaces dates back to Gibbs ${ }^{1}$ and some 40 years ago a statistical mechanical treatment of the influence of curvature was given by the work of Kirkwood and Buff., ${ }^{2,3}$ Due to the introduction of the rigidity constants and the spontaneous curvature of an interface by Helfrich ${ }^{4}$ in 1973, the importance of the influence of curvature has, in recent years, been more clearly recognized. An important formula in the description of spherical surfaces is the Laplace equation ${ }^{5}$ which relates the pressure difference between both sides of the surface of the droplet to the surface tension $\sigma$ and the equimolar radius of the droplet $R$,

$$
\Delta p=\frac{2 \sigma}{R},
$$

where $\Delta p=p_{l}-p_{g}$ with $p_{l}$ and $p_{g}$ the pressure in the liquid and gas region. For droplets with small radii, Eq. (1.1) is only approximately valid and this formula should be seen as the lowest-order term in an expansion of $\Delta p$ in the reciprocal radius. The first-order correction to Eq. (1.1) defines the socalled Tolman length $\delta$ (Ref. 6)

$$
\Delta p=\frac{2 \sigma}{R}\left(1-\frac{\delta}{R}\right) \text {. }
$$

If the droplet is large enough compared to the thickness of the interfacial region, the values of $p_{l}$ and $p_{g}$ may be identified as the "bulk" pressures in the center of the droplet and far outside the droplet, respectively. If the droplet is smaller one may question the possibility to give a satisfactory definition of the pressure in the center of the droplet as a bulk pressure. In order to uniquely define the pressure difference even for small droplets, $\Delta p$ is defined as the pressure difference between a bulk liquid and bulk gas calculated from the given temperature and chemical potential. Important in this context is that $\Delta p$ is a unique function of $\mu$ and $T$ and does not depend on the choice of the position of the dividing surface. Such a different choice of the position of the dividing surface and thus the value of the radius $R$, rearranges the terms on the right-hand side of Eq. (1.2) but does not change $\Delta p$. A lot of effort has been made to calculate $\delta$ using molecular dynamics (MD) simulations ${ }^{7,8}$ of Lennard-Jones fluids but up till now no consensus has been reached whether $\delta$ has a finite or a zero value. The most recent and extensive calculations ${ }^{8}$ indicate that within the error $\delta$ cannot be distinguished from zero. The first statistical mechanical expression for $\delta$ was given by Kirkwood and Buff ${ }^{2}$ who argued that, as the surface tension is given by the zeroth moment of the excess pressure in the interfacial region, $\delta$ should be given by the first moment. On the basis of this consideration they proposed a microscopic expression for $\delta$ found from their formula for $\sigma$ by multiplying the integrand with the distance to the surface.

An alternative route to obtain expressions for $\sigma$ and $\delta$ is to use a microscopic expression for the pressure tensor in an inhomogeneous system. The first such expression for the pressure tensor was given by Irving and Kirkwood. ${ }^{9}$ Later, Schofield and Henderson ${ }^{10}$ pointed out that it is not possible to define the microscopic pressure tensor uniquely and they proposed a more general expression which reflects this nonunique nature. Recently, the merit of their alternative definitions has been the subject of some discussion. ${ }^{11-13}$ For an interface one may calculate the surface tension as an integral over the normal coordinate of the pressure difference parallel and orthogonal to the surface. Similarly, one obtains an expression for $\delta$ by integrating the pressure difference times the normal coordinate. A brief review of some of these matters is given in Sec. II.

In a previous paper ${ }^{14}$ expressions were derived using statistical mechanics for the surface tension $\sigma(R)$ and curvature term $C(R)$, i.e., the partial derivative of the free energy with respect to the radius $R$, of a spherical liquid-vapor interface. The coefficients in an expansion to second order in the reciprocal radius of the interface of $\sigma(R)$ and $C(R)$ are related to the surface tension of the flat interface $\sigma$, the spontaneous curvature of the interface $C_{0}$, the rigidity constant 
associated with Gaussian curvature $\bar{k}$, and the rigidity constant of bending $k$. The resulting formulas give these coefficients as expressions in terms of integrals of the pair density $\rho^{(2)}\left(\mathbf{r}_{1}, \mathbf{r}_{2}\right)$ and the derivative of the interaction potential $u^{\prime}(r)$. The Laplace equation in terms of $\sigma(R)$ and $C(R)$ can be derived from an analysis of the total change in free energy under changing the radius of the droplet. ${ }^{5}$ The resulting expression can again be expanded in powers of the reciprocal radius

$$
\Delta p=\frac{2 \sigma(R)}{R}+C(R)=\frac{2 \sigma}{R}+2 k C_{0}\left(\frac{1}{R}\right)^{2}
$$

From Eqs. (1.2) and (1.3) it is clear that Tolman's length is given by

$$
\delta=-k C_{0} / \sigma
$$

The purpose of this paper is to compare the microscopic formula for $\Delta p$ to second order in the reciprocal radius, derived in a previous paper, ${ }^{14}$ to the one obtained using the microscopic expression for the pressure tensor of the spherical interface. In particular, we want to clarify the somewhat confusing discussion about its proper definition by establishing to what extent the expressions found using the various pressure tensors agree with the sum rules obtained exactly. In Sec. III we find that the expression for $\Delta p$ found using the Irving-Kirkwood expression for the pressure tensor is identical to the one found using the rigorous microscopic expressions for $\sigma(R)$ and $C(R)$. A similar analysis using the Schofield and Henderson definition of the pressure tensor for an alternative choice of the integration contour, which features in their expression, is done in Appendix $\mathrm{A}$ and gives an incorrect result for $\Delta p$ and in particular for the Tolman length. The alternative contour we use reduces to the one which may be used to obtain the expression for the pressure tensor given by Harasima ${ }^{15}$ for a flat interface. Furthermore we find, see Sec. IV and Appendix B, that both expressions for Tolman's length mentioned earlier are incorrect. The underlying reason is the fact that the Tolman length differs from the distance between the mechanical surface of tension and the Gibbs dividing surface. It also follows from the MD results for droplets of Nijmeijer $e t a l^{8}$ that the distance between the mechanical surface of tension and the Gibbs dividing surface is considerably different from Tolman's length which, in fact, is found to be equal to zero within the error. The numerical value for the location of the mechanical surface of tension and the fact that Tolman's length is zero is further discussed using an approximate expression for the pair density. It should be noted that Tolman uses a different definition of the surface of tension which he defines by ${ }^{6}$ $\Delta p \equiv 2 \sigma_{s} / R_{s}=2 \sigma\left(R_{s}+2 \delta\right)^{-1}$. Comparing with Eq. (1.2) one then sees that $R_{s}-R=-\delta$ so that its distance to the equimolar surface is given by (minus) the Tolman length. That these two definitions may lead to confusion is most apparent in the commonly accepted statement that the location of the mechanical surface of tension relative to the equimolar surface, will give the Tolman length ${ }^{2}$ which, as we will show, is not correct. A summary of results is given in Sec. V.

\section{THE LAPLACE EQUATION}

We briefly review the most important results of the thermodynamic analysis of a spherical interface which is used to derive the Laplace equation which includes the contribution due to the curvature term. Using Helfrich's expression for the curvature dependent surface tension we then proceed to give the pressure difference to second order in the curvature. In the last part of this section we also present the derivation of $\Delta p$ using the pressure tensor. Both routes to obtain $\Delta p$ are well known and we refer the reader to the excellent reviews of Rowlinson and Widom ${ }^{5}$ and Ono and $\mathrm{Kondo}^{16}$ for a more extensive discussion.

The change in excess free energy $F^{s}$ of a spherical interface with radius $R$ is given by

$$
d F^{s}=-S^{s} d T+\mu d N^{s}+\sigma(R) d A+C(R) A d R,
$$

where $T$ is the temperature, $S^{s}$ is the excess entropy, $\mu$ is the chemical potential, $N^{s}$ is the excess number density, $\sigma(R)$ is the radius dependent surface tension, $A$ the surface area, and $C(R)$ is the coefficient of the curvature term. The excess of an extensive quantity is defined as the difference between the total amount of that quantity and the integral over the dividing surface extrapolated bulk densities of this quantity. The excess number density is thus given by

$$
N^{s}=N-\int d V\left(\rho_{l} \theta_{l}+\rho_{g} \theta_{g}\right)
$$

where $\rho_{l}$ and $\rho_{g}$ are the number densities in the liquid and gas phase, the characteristic function $\theta_{l}$ is defined to be equal to one on the liquid side of the dividing surface and zero on the gas side, furthermore $\theta_{l}+\theta_{g} \equiv 1$. From this equation it is clear that $N^{s}$ depends on the choice of the position of the dividing surface. For simplicity we will take the Gibbs dividing surface, or equimolar surface, for which $N^{s} \equiv 0$, throughout this paper. We stress that other possible choices of the position of the dividing surface lead to the same results for all measurable quantities as, for instance, the pressure difference $\Delta p$. With $N^{s}=0$, Eq. (2.1) reduces to

$$
d F^{s}=-S^{s} d T+\sigma(R) d A+C(R) A d R .
$$

Integration over extensive variables yields

$$
F^{s}=\sigma(R) A .
$$

The resulting Maxwell equation relates $\sigma(R)$ and $C(R)$ by

$$
C(R)=\left.\frac{\partial \sigma(R)}{\partial R}\right|_{T} .
$$

The Laplace equation for a spherical interface is most conveniently derived by considering the change in the total free energy of the system due to a notional change $e^{5}$ of the radius

$$
\begin{aligned}
{[d F]=} & -p_{l}\left[d V_{l}\right]-p_{g}\left[d V_{g}\right] \\
& +\sigma(R)[d A]+C(R) A[d R] .
\end{aligned}
$$

The square brackets denote a change as a consequence of a different choice of the radius and not a physical change in the radius of the droplet. Of course the free energy cannot de- 
pend on this choice, $[d F]=0$, so one finds from Eq. (2.6) that

$$
\Delta p=\frac{2 \sigma(R)}{R}+C(R),
$$

where we have used that $\left[d V_{l}\right]=-\left[d V_{g}\right]=A[d R]$ and $[d A]=(2 A / R)[d R]$. This equation is the Laplace equation generalized to include higher-order curvature corrections.

In general, the surface tension depends on the two principal radii of curvature $R_{1}$ and $R_{2}$ of the surface. Helfrich ${ }^{2}$ introduced the following expansion of the surface tension to second order in the inverse radii:

$$
\sigma\left(R_{1}, R_{2}\right)=f_{0}+\frac{k}{2}\left(\frac{1}{R_{1}}+\frac{1}{R_{2}}+C_{0}\right)^{2}+\bar{k} \frac{1}{R_{1} R_{2}},
$$

where $C_{0}$ is called the spontaneous curvature, $k$ is the rigidity constant of bending, and $\bar{k}$ is the rigidity constant associated with Gaussian curvature. The coefficient $f_{0}$ is related to the surface tension of the planar interface by $f_{0}+(k / 2) C_{0}^{2}=\sigma$. One may question the possibility to expand the surface tension in the curvatures. Arguments have been given which imply that $\sigma\left(R_{1}, R_{2}\right)$ is not an analytic function. In the expansion this shows up as a logarithmic divergence in the microscopic expressions for the expansion coefficients. In the previous paper we discussed this matter and concluded that as long as the interaction potential decays as $1 / r,{ }^{7}$ or faster, the expansion is correct to second order. Use of Helfrich's formula for the surface free-energy yields for the spherical interface where $R_{1}=R_{2}=R$

$$
\begin{aligned}
& \sigma(R)=\sigma+2 k C_{0} \frac{1}{R}+(2 k+\bar{k})\left(\frac{1}{R}\right)^{2}, \\
& C(R)=\left.\frac{\partial \sigma}{\partial R}\right|_{T}=-2 k C_{0}\left(\frac{1}{R}\right)^{2}-2(2 k+\bar{k})\left(\frac{1}{R}\right)^{3} .
\end{aligned}
$$

When we insert these expressions into the Laplace equation, Eq. (2.7), we find

$$
\Delta p=\frac{2 \sigma}{R}+2 k C_{0}\left(\frac{1}{R}\right)^{2} .
$$

Notice that the contribution due to the second-order term in the expansion of the surface tension vanishes. This has lead several authors in the past to conclude that such an expansion in the reciprocal radius is not possible beyond first order for the spherical interface. ${ }^{5,11}$ As the surface tension (excess free energy) contains these contributions and they not only contribute to the pressure difference for all other surface shapes but it is also possible to derive exact expressions for the coefficients $k$ and $\bar{k}$ analogous to the Kirkwood-Buff formula for the surface tension, ${ }^{14}$ we do not agree with this point of view.

We now turn to a description of an inhomogeneous system using a position-dependent pressure tensor. Due to the spherical symmetry of the system this position-dependent pressure tensor has only two independent components, normal and tangential to the interface

$$
\mathbf{p}(\mathbf{r})=p_{N}(r) \hat{e}_{r} \hat{e}_{r}+p_{T}(r)\left(\mathbf{1}-\hat{e}_{r} \hat{e}_{r}\right),
$$

where $\mathbf{1}$ is the unit tensor, $\hat{e}_{r} \equiv \mathbf{r} /|\mathbf{r}|$ is the unit vector in the direction $\mathbf{r}$ and $r \equiv|\mathbf{r}|$. Far from the surface the normal and tangential component of the pressure tensor become identical and reduces the pressure tensor to a constant scalar pressure. When no external field is present the requirement that the droplet is in mechanical equilibrium imposes the following condition on the pressure tensor:

$$
\boldsymbol{\nabla} \cdot \mathbf{p}(\mathbf{r})=\mathbf{0}
$$

Inserting Eq. (2.11) in this equation yields the following relation between the components of the pressure tensor

$$
p_{N}^{\prime}(r)=\frac{2}{r}\left[p_{T}(r)-p_{N}(r)\right],
$$

where the prime indicates a differentiation with respect to its argument. Integration of the above expression from the inside to the outside of the droplet yields

$$
\Delta p=2 \int_{0}^{\infty} d r \frac{1}{r}\left[p_{N}(r)-p_{T}(r)\right]
$$

The local pressure tensor at a certain point $r$ in an inhomogeneous fluid comprises two terms. The first term is isotropic and equals the familiar expression for the pressure in an ideal gas. The second term is due to pair interaction between the particles. Irving and Kirkwood give the formula ${ }^{9}$

$$
\begin{aligned}
\mathbf{p}(\mathbf{r})= & k_{B} T \rho(\mathbf{r}) \mathbf{1}-\frac{1}{2} \int d \mathbf{r}_{12} \int_{0}^{1} d \alpha \frac{\mathbf{r}_{12} \mathbf{r}_{12}}{r_{12}} u^{\prime}\left(r_{12}\right) \\
& \times \rho_{s}^{(2)}\left(\mathbf{r}-\alpha \mathbf{r}_{12}, \mathbf{r}+(1-\alpha) \mathbf{r}_{12}\right),
\end{aligned}
$$

where $r_{12} \equiv\left|\mathbf{r}_{12}\right|, \rho(\mathbf{r})$ is the number density and $\rho_{s}^{(2)}\left(\mathbf{r}_{1}, \mathbf{r}_{2}\right)$ is the pair density of the spherical surface. On the basis of the nonunique nature of the pressure tensor it has been argued by Schofield and Henderson ${ }^{10}$ that the integration over $\alpha$ in Eq. (2.15) can be replaced by a line integral along an arbitrary path from 0 to $\mathbf{r}_{12}$

$$
\begin{aligned}
p_{\alpha \beta}(\mathbf{r})= & k_{B} T \rho(\mathbf{r}) \delta_{\alpha \beta}-\frac{1}{2} \int d \mathbf{r}_{12} \int d l_{\beta} \frac{r_{12, \alpha}}{r_{12}} u^{\prime}\left(r_{12}\right) \\
& \times \rho_{s}^{(2)}\left(\mathbf{r}-\mathbf{l}, \mathbf{r}+\mathbf{r}_{12}-\mathbf{I}\right),
\end{aligned}
$$

where $\alpha, \beta=x, y, z$ and $\delta_{\alpha \beta}$ is the Kronecker delta. This is an equivalent expression for the pressure tensor in the sense that its divergence is said ${ }^{10}$ to be equal to the divergence of the expression in Eq. (2.15). Recently, Baus and Lovett ${ }^{11}$ argued that one may impose an additional condition on the pressure tensor which eliminates the ambiguity in the definition of the pressure tensor. It is tentatively concluded that the Irving-Kirkwood definition of the pressure tensor given by the straight line integral in Eq. (2.15), is the only correct choice. ${ }^{17}$ As it has subsequently been argued by Rowlinson $^{12}$ that the Baus-Lovett condition on the pressure tensor implies that the condition leads to a vanishing surface tension, an argument which, in our opinion, has not really been convincingly refuted by Baus and Lovett, ${ }^{13}$ this matter is as yet unclear.

\section{CALCULATION OF THE LAPLACE EQUATION USING THE PRESSURE TENSOR}

In this section we calculate the pressure difference from Eq. (2.15) using the Irving-Kirkwood expression from Eq. 
(2.14) to second order in the reciprocal radius. The normal and tangential component of the pressure tensor are then given by

$$
\begin{aligned}
p_{N}(r)= & k_{B} T \rho(r)-\frac{1}{2} \int d \mathbf{r}_{12} \int_{0}^{1} d \alpha \frac{\left(\mathbf{r}_{12} \cdot \hat{e}_{r}\right)^{2}}{r_{12}} u^{\prime}\left(r_{12}\right) \\
& \times \rho_{s}^{(2)}\left(\mathbf{r}-\alpha \mathbf{r}_{12}, \mathbf{r}+(1-\alpha) \mathbf{r}_{12}\right) \\
p_{T}(r)= & k_{B} T \rho(r) \\
& -\frac{1}{2} \int d \mathbf{r}_{12} \int_{0}^{1} d \alpha \frac{r_{12}^{2}-\left(\mathbf{r}_{12} \cdot \hat{e}_{r}\right)^{2}}{2 r_{12}} u^{\prime}\left(r_{12}\right) \\
& \times \rho_{s}^{(2)}\left(\mathbf{r}-\alpha \mathbf{r}_{12}, \mathbf{r}+(1-\alpha) \mathbf{r}_{12}\right) .
\end{aligned}
$$

For convenience we choose the $z$ axis along $\mathbf{r}$ so that $\mathbf{r}=z \hat{z}$ and $\hat{e}_{r}=\hat{z}$. The difference between the normal and tangential component of the pressure tensor is then given by

$$
\begin{aligned}
{\left[p_{N}-p_{T}\right](z)=} & \frac{1}{4} \int d \mathbf{r}_{12} \int_{0}^{1} d \alpha u^{\prime}(r) r\left(1-3 s^{2}\right) \\
& \times \rho_{s}^{(2)}\left(z \hat{z}-\alpha \mathbf{r}_{12}, z \hat{z}+(1-\alpha) \mathbf{r}_{12}\right)
\end{aligned}
$$

with $s \equiv \cos \theta_{12}$ and $r \equiv r_{12}$. Due to the spherical symmetry of the system the pair density can be written as

$$
\rho_{s}^{(2)}\left(\mathbf{r}_{1}, \mathbf{r}_{2}\right)=\rho_{s}^{(2)}\left(\left|\mathbf{r}_{1}\right|,\left|\mathbf{r}_{2}\right|, r\right)
$$

so that the full expression for the difference between the normal and tangential component of the pressure tensor is equal to

$$
\begin{aligned}
{\left[p_{N}-p_{T}\right](z)=} & \frac{1}{4} \int d \mathbf{r}_{12} \int_{0}^{1} d \alpha u^{\prime}(r) r\left(1-3 s^{2}\right) \\
& \times \rho_{s}^{(2)}\left(\left|z \hat{z}-\alpha \mathbf{r}_{12}\right|,\left|z \hat{z}+(1-\alpha) \mathbf{r}_{12}\right|, r\right) .
\end{aligned}
$$

We now make an expansion to second order in the reciprocal radius of the droplet of the integrand in Eq. (3.4). The expression for the pressure difference $\Delta p$ to second order can then be obtained by integrating the resulting expression over $z$, cf. Eq. (2.14). The resulting formula for $\Delta p$ from this analysis can be found in Eq. (3.13). If one is more interested in the discussion rather than in the explicit derivation of this result, it is possible to continue reading after Eq. (3.13).

We now proceed to make an expansion of the pair density in the ratio of the intermolecular distance and the radius of the droplet, i.e., $r / R$, to second order. As was discussed before, the use of such an expansion in the integrand is possible when the interaction potential is sufficiently short ranged, cf. Ref. 14 for a more extensive discussion of this point. The arguments of the pair density, $\left|z \hat{z}-\alpha \mathbf{r}_{12}\right|$ and $\left|z \hat{z}+(1-\alpha) \mathbf{r}_{12}\right|$, are up to second order in $r / z$ given by

$$
\begin{aligned}
\left|z \hat{z}-\alpha \mathbf{r}_{12}\right|= & z-\alpha s r+\frac{1}{2 z} \alpha^{2} r^{2}\left(1-s^{2}\right) \\
& +\frac{1}{2}\left(\frac{1}{z}\right)^{2} \alpha^{3} r^{3} s\left(1-s^{2}\right),
\end{aligned}
$$

$$
\begin{aligned}
\mid z \hat{z}+ & (1-\alpha) \mathbf{r}_{12} \mid \\
= & z+(1-\alpha) s r+\frac{1}{2 z}(1-\alpha)^{2} r^{2}\left(1-s^{2}\right) \\
& -\frac{1}{2}\left(\frac{1}{z}\right)^{2}(1-\alpha)^{3} r^{3} s\left(1-s^{2}\right) .
\end{aligned}
$$

It should be realized that the integrand in Eq. (3.4) only differs from zero when $z$ lies in the interfacial region, i.e., $z \approx R$, so that the expansion in $r / z$ is, in fact, an expansion in $r / R$. We now want to expand the pair density itself in $r / R$. This is done by making an expansion of the pair density around the pair density with the first and second argument replaced by the first two terms in Eq. (3.5),

$$
\begin{aligned}
& \rho_{s}^{(2)}\left(\left|z \hat{z}-\alpha \mathbf{r}_{12}\right|,\left|z \hat{z}+(1-\alpha) \mathbf{r}_{12}\right|, r\right) \\
& =\rho_{s}^{(2)}(z-\alpha s r, z+(1-\alpha) s r, r)+\rho_{1}^{(2)}\left[\frac{1}{2 z} \alpha^{2} r^{2}\left(1-s^{2}\right)+\frac{1}{2}\left(\frac{1}{z}\right)^{2} \alpha^{3} r^{3} s\left(1-s^{2}\right)\right] \\
& \quad+\rho_{2}^{(2)}\left[\frac{1}{2 z}(1-\alpha)^{2} r^{2}\left(1-s^{2}\right)-\frac{1}{2}\left(\frac{1}{z}\right)^{2}(1-\alpha)^{3} r^{3} s\left(1-s^{2}\right)\right] \\
& \quad+\frac{1}{2} \rho_{11}^{(2)}\left(\frac{1}{2 z} \alpha^{2} r^{2}\left(1-s^{2}\right)\right)^{2}+\rho_{12}^{(2)}\left(\frac{1}{2 z} \alpha^{2} r^{2}\left(1-s^{2}\right)\right)\left(\frac{1}{2 z}(1-\alpha)^{2} r^{2}\left(1-s^{2}\right)\right)+\frac{1}{2} \rho_{22}^{(2)}\left(\frac{1}{2 z}(1-\alpha)^{2} r^{2}\left(1-s^{2}\right)\right)^{2}
\end{aligned}
$$

The subindices of the pair density indicate partial differentiation with respect to its first or second argument, e.g., $\rho_{1}^{(2)}=\left.(\partial / \partial t) \rho_{s}^{(2)}[t, z+(1-\alpha) s r, r]\right|_{t=z-\alpha s r}$. We furthermore did not write the explicit dependence of the pair density on its arguments for the higher order derivatives. The proper explicit dependence is given in the first term in the above expansion. It should be noted that $\rho_{1}^{(2)}$ and $\rho_{2}^{(2)}$ are of order $1 / r$ and $\rho_{11}^{(2)}, \rho_{12}^{(2)}$, and $\rho_{22}^{(2)}$ of order $1 / r^{2}$ relative to $\rho_{s}^{(2)}$. As a result Eq. (3.6) is the proper way to expand the pair density so that higher-order terms in the above expansion correspond to higher powers in $r / R$; this would not be the case if one expands around $z$. We write the partial differentiation with respect to the first and second argument in terms of a differentiation with respect to $s$ and $\alpha$ 


$$
\begin{aligned}
& \rho_{1}^{(2)}=\frac{-1}{r} \frac{d}{d s} \rho_{s}^{(2)}-\frac{(1-\alpha)}{r s} \frac{d}{d \alpha} \rho_{s}^{(2)}, \\
& \rho_{2}^{(2)}=\frac{1}{r} \frac{d}{d s} \rho_{s}^{(2)}-\frac{\alpha}{r s} \frac{d}{d \alpha} \rho_{s}^{(2)}, \\
& \rho_{11}^{(2)}=\frac{1}{r^{2}} \frac{d^{2}}{d s^{2}} \rho_{s}^{(2)}+\frac{2(1-\alpha)}{r^{2} s} \frac{d^{2}}{d s d \alpha} \rho_{s}^{(2)}+\frac{(1-\alpha)^{2}}{r^{2} s^{2}} \frac{d^{2}}{d \alpha^{2}} \rho_{s}^{(2)}-\frac{2(1-\alpha)}{r^{2} s^{2}} \frac{d}{d \alpha} \rho_{s}^{(2)}, \\
& \rho_{12}^{(2)}=\frac{-1}{r^{2}} \frac{d^{2}}{d s^{2}} \rho_{s}^{(2)}+\frac{(2 \alpha-1)}{r^{2} s} \frac{d^{2}}{d s d \alpha} \rho_{s}^{(2)}+\frac{\alpha(1-\alpha)}{r^{2} s^{2}} \frac{d^{2}}{d \alpha^{2}} \rho_{s}^{(2)}+\frac{(1-2 \alpha)}{r^{2} s^{2}} \frac{d}{d \alpha} \rho_{s}^{(2)}, \\
& \rho_{22}^{(2)}=\frac{1}{r^{2}} \frac{d^{2}}{d s^{2}} \rho_{s}^{(2)}-\frac{2 \alpha}{r^{2} s} \frac{d^{2}}{d s d \alpha} \rho_{s}^{(2)}+\frac{\alpha^{2}}{r^{2} s^{2}} \frac{d^{2}}{d \alpha^{2}} \rho_{s}^{(2)}+\frac{2 \alpha}{r^{2} s^{2}} \frac{d}{d \alpha} \rho_{s}^{(2)} .
\end{aligned}
$$

Using Eqs. (3.6) and (3.7), to second order in $r / R$, Eq. (3.4) becomes equal to

$$
\begin{aligned}
{\left[p_{N}-p_{T}\right](z)=} & \frac{1}{4} \int d \mathbf{r}_{12} \int_{0}^{1} d \alpha u^{\prime}(r) r\left(1-3 s^{2}\right)\left\{1+\frac{r^{2}\left(1-s^{2}\right)}{2 z}\right. \\
& \times\left((1-2 \alpha) \frac{1}{r} \frac{d}{d s}+\frac{\alpha(\alpha-1)}{r s} \frac{d}{d \alpha}-\frac{s}{z}\left(1-3 \alpha+3 \alpha^{2}\right) \frac{d}{d s}+\frac{1}{z} \alpha(1-2 \alpha)(1-\alpha) \frac{d}{d \alpha}\right) \\
& +\frac{r^{2}\left(1-s^{2}\right)^{2}}{8 z^{2}}\left[(1-2 \alpha)^{2} \frac{d^{2}}{d s^{2}}+2 \alpha(1-2 \alpha)(1-\alpha) \frac{1}{s} \frac{d}{d \alpha}\left(\frac{1}{s}-\frac{d}{d s}\right)\right. \\
& \left.\left.+\frac{\alpha^{2}(1-\alpha)^{2}}{s^{2}} \frac{d^{2}}{d \alpha^{2}}\right]\right\} \rho_{s}^{(2)}(z-\alpha s r, z+(1-\alpha) s r, r) .
\end{aligned}
$$

After partial integration over $\alpha$ and $s$ this reduces to

$$
\begin{aligned}
{\left[p_{N}-p_{T}\right](z)=} & \frac{1}{4} \int d \mathbf{r}_{12} \int_{0}^{1} d \alpha u^{\prime}(r) r \rho_{s}^{(2)}(z-\alpha s r, z+(1-\alpha) s r, r)\left[\left(1-3 s^{2}\right)+\frac{r}{2 s z}(1-2 \alpha)\left(1+4 s^{2}-9 s^{4}\right)\right. \\
& +\frac{r^{2}}{4 s^{2} z^{2}}\left(\left(1-3 \alpha+3 \alpha^{2}\right)\left(2 s^{2}-24 s^{4}+30 s^{6}\right)-(1-2 \alpha)^{2}\left(5 s^{2}-42 s^{4}+45 s^{6}\right)\right. \\
& \left.\left.+\left(1-6 \alpha+6 \alpha^{2}\right)\left(1+3 s^{2}-13 s^{4}+9 s^{6}\right)\right)\right]
\end{aligned}
$$

Writing $z \equiv R+z_{1}$, where $R$ is the equimolar radius and using Eq. (2.14) together with the above equation, we arrive at the following expression for the pressure difference across the spherical drop:

$$
\begin{aligned}
\Delta p= & \frac{1}{2 R} \int d z_{1} \int d \mathbf{r}_{12} \int_{0}^{1} d \alpha u^{\prime}(r) r \rho_{s}^{(2)}\left(R+z_{1}-\alpha s r, R+z_{1}+(1-\alpha) s r, r\right) \\
& \times\left[\left(1-3 s^{2}\right)\left(1-\frac{z_{1}}{R}+\frac{z_{1}^{2}}{R^{2}}\right)+\frac{1}{R} \frac{r}{2 s}(1-2 \alpha)\left(1+4 s^{2}-9 s^{4}\right)\left(1-\frac{2 z_{1}}{R}\right)\right. \\
& +\left(\frac{1}{R}\right)^{2} \frac{r^{2}}{4 s^{2}}\left(\left(1-3 \alpha+3 \alpha^{2}\right)\left(2 s^{2}-24 s^{4}+30 s^{6}\right)\right. \\
& \left.\left.-(1-2 \alpha)^{2}\left(5 s^{2}-42 s^{4}+45 s^{6}\right)+\left(1-6 \alpha+6 \alpha^{2}\right)\left(1+3 s^{2}-13 s^{4}+9 s^{6}\right)\right)\right] .
\end{aligned}
$$

The first and second argument of the pair density measure the distance from the center of the droplet. Notationally it is more convenient to take the distance to the equimolar surface as a parameter for the pair density so that from now on $R$ drops from the expressions for the first and second argument in the pair density. In the expression for $\Delta p$ in Eq. (3.1) we now shift the integration over $z_{1}$ over a distance $\alpha s r$, i.e. $z_{1} \rightarrow z_{1}+\alpha s r$. Care has to be taken in doing this as the boundaries in this shift are itself integration parameters. One therefore has to subtract from the integrand in Eq. (3.10) the extrapolated integrand from the bulk regions. This is done by subtracting the extrapolated bulk pair density $\rho_{s, i, g}^{(2)}\left(z_{1}, r\right)$ which equals the liquid pair density $\rho_{s, l}^{(2)}(r)$ for $z_{1}<0$ and the gas pair density $\rho_{s: g}^{(2)}(r)$ for $z_{1}>0$, from the pair density in the interfacial region. Equation (3.10) then can be written as a sum of two terms:

$$
\begin{aligned}
\Delta p= & \frac{1}{2 R} \int d z_{1} \int d \mathbf{r}_{12} \int_{0}^{1} d \alpha u^{\prime}(r) r\left[\rho_{s}^{(2)}\left(z_{1}, z_{1}+s r, r\right)-\rho_{s, l, g}^{(2)}\left(z_{1}, r\right)\right] \\
& \times\left\{\left(1-3 s^{2}\right)\left[1-\frac{1}{R}\left(z_{1}+\alpha s r\right)+\left(\frac{1}{R}\right)^{2}\left(z_{1}+\alpha s r\right)^{2}\right]\right.
\end{aligned}
$$




$$
\begin{aligned}
& +\frac{1}{R} \frac{r}{2 s}(1-2 \alpha)\left(1+4 s^{2}-9 s^{4}\right)\left(1-\frac{2}{R}\left(z_{1}+\alpha s r\right)\right)+\frac{r^{2}}{4 s^{2} R^{2}}\left[\left(1-3 \alpha+3 \alpha^{2}\right)\left(2 s^{2}-24 s^{4}+30 s^{6}\right)\right. \\
& \left.\left.-(1-2 \alpha)^{2}\left(5 s^{2}-42 s^{4}+45 s^{6}\right)+\left(1-6 \alpha+6 \alpha^{2}\right)\left(1+3 s^{2}-13 s^{4}+9 s^{6}\right)\right]\right\} \\
& +\frac{1}{2 R} \int d z_{1} \int d \mathbf{r}_{12} \int_{0}^{1} d \alpha u^{\prime}(r) r\left[\rho_{s, l, g}^{(2)}\left(z_{1}, r\right)-\rho_{s, l, g}^{(2)}\left(z_{1}+\alpha s r, r\right)\right]\left\{( 1 - 3 s ^ { 2 } ) \left[1-\frac{1}{R}\left(z_{1}+\alpha s r\right)\right.\right. \\
& \left.+\left(\frac{1}{R}\right)^{2}\left(z_{1}+\alpha s r\right)^{2}\right]+\frac{1}{R} \frac{r}{2 s}(1-2 \alpha)\left(1+4 s^{2}-9 s^{4}\right)\left(1-\frac{2}{R}\left(z_{1}+\alpha s r\right)\right) \\
& +\frac{r^{2}}{4 s^{2} R^{2}}\left(\left(1-3 \alpha+3 \alpha^{2}\right)\left(2 s^{2}-24 s^{4}+30 s^{6}\right)-(1-2 \alpha)^{2}\left(5 s^{2}-42 s^{4}+45 s^{6}\right)\right. \\
& \left.\left.+\left(1-6 \alpha+6 \alpha^{2}\right)\left(1+3 s^{2}-13 s^{4}+9 s^{6}\right)\right)\right\} .
\end{aligned}
$$

The second term in Eq. (3.11) vanishes after subsequently integrating over $z_{1}, \alpha$, and $s$,

$$
\begin{aligned}
\frac{1}{2 R} \int d \mathbf{r}_{12} \int_{0}^{1} d \alpha u^{\prime}(r) r^{2}\left[\rho_{s, g}^{(2)}(r)-\rho_{s, l}^{(2)}(r)\right]\left\{-\alpha s\left(1-3 s^{2}\right)\left[1-\frac{\alpha s r}{2 R}+\frac{1}{3}\left(\frac{\alpha s r}{R}\right)^{2}\right]\right. \\
\quad-\frac{\alpha r}{2 R}(1-2 \alpha)\left(1+4 s^{2}-9 s^{4}\right)\left(1-\frac{\alpha s r}{R}\right)-\frac{\alpha r^{2}}{4 s R^{2}}\left[\left(1-3 \alpha+3 \alpha^{2}\right)\left(2 s^{2}-24 s^{4}+30 s^{6}\right)\right. \\
\left.\left.\quad-(1-2 \alpha)^{2}\left(5 s^{2}-42 s^{4}+45 s^{6}\right)+\left(1-6 \alpha+6 \alpha^{2}\right)\left(1+3 s^{2}-13 s^{4}+9 s^{6}\right)\right]\right\} \\
\quad=\frac{1}{4 R} 2 \pi \int d r \int_{-1}^{1} d s u^{\prime}(r) r^{4}\left[\rho_{s: 8}^{(2)}(r)-\rho_{s, l}^{(2)}(r)\right]\left(\left(3 s^{3}-s\right)+\frac{r}{6 R}\left(1+6 s^{2}-15 s^{4}\right)+\frac{s r^{2}}{3 R^{2}}\left(1+17 s^{2}-3 s^{4}\right)\right)=0
\end{aligned}
$$

In the first term of Eq. (3.11), the integration over $\alpha$ can be carried out so that we are finally left with the following expression for the pressure difference which follows from the Irving-Kirkwood choice for the pressure tensor:

$$
\Delta p=\frac{1}{2 R} \int d z_{1} \int d \mathbf{r}_{12} u^{\prime}(r) r \rho_{s}^{(2)}\left(z_{1}, z_{2}, r\right)\left[\left(1-3 s^{2}\right)\left(1-\frac{z_{1}+z_{2}}{2 R}+\frac{z_{1} z_{2}}{R^{2}}\right)+\frac{r^{2} s^{2}}{2 R^{2}}\left(3-5 s^{2}\right)\right],
$$

where $z_{2} \equiv z_{1}+s r$.

In a previous paper it has been rigorously shown that ${ }^{14}$

$$
\begin{aligned}
& \sigma(R)=\frac{1}{4} \int d z_{1} \int d \mathbf{r}_{12} u^{\prime}(r) r \rho_{s}^{(2)}\left(z_{1}, z_{2}, r\right)\left[\left(1-3 s^{2}\right)\left(1+\frac{z_{1} z_{2}}{R^{2}}\right)+\frac{r^{2} s^{2}}{3 R^{2}}\left(3-5 s^{2}\right)\right], \\
& C(R)=\frac{1}{4 R} \int d z_{1} \int d \mathbf{r}_{12} u^{\prime}(r) r \rho_{s}^{(2)}\left(z_{1}, z_{2}, r\right)\left[-\left(1-3 s^{2}\right)\left(\frac{z_{1}+z_{2}}{R}\right)+\frac{r^{2} s^{2}}{3 R^{2}}\left(3-5 s^{2}\right)\right] .
\end{aligned}
$$

If one substitutes these expressions into the Laplace equation (2.7) one obtains Eq. (3.13) for the pressure difference directly. This shows that the Irving-Kirkwood expression for the pressure tensor leads to a correct expression for the pressure difference to third order in the inverse equimolar radius. Using a path different from the straight line integral in expression (2.16) for the pressure tensor of Schofield and Henderson, we calculate the resulting pressure difference in Appendix A. The path we choose leads to the so-called Harasima expression for the pressure tensor ${ }^{15}$ of a planar interface. The resulting expression for $\Delta p$ of a curved interface, however, differs from the expression given in Eq. (3.13). Although the zeroth-order term in the expansion of $\Delta p$, which gives the surface tension of the flat interface, is the same for both routes, the first-order terms are different. It is clear that even though there may be some ambiguity in the definition of the pressure tensor the pressure difference, being a measurable quantity, cannot depend on the choice of the pressure tensor. It must therefore be concluded that the
Schofield-Henderson expression is not correct for an arbitrary choice of the contour.

\section{COMPARISON WITH PREVIOUS RESULTS AND COMPUTER SIMULATIONS.}

In this section we want to compare previous derived expressions for the radius dependent surface tension and curvature term with the rigorous expressions given in Eq. (3.14). In particular, we will compare the different expressions that have appeared in the literature for Tolman's length. In Ref. 14 we derived, on the basis of the expression for $C(R)$ given in Eq. (3.14), an expression for $k C_{0}$ which gives, upon substitution in Eq. (1.4), the following formula for Tolman's length:

$$
\begin{aligned}
\delta= & \frac{-1}{8 \sigma} \int d z_{1} \int d \mathbf{r}_{12} u^{\prime}(r) r\left(1-3 s^{2}\right) \\
& \times\left(z_{1}+z_{2}\right) \rho_{f}^{(2)}\left(z_{1}, z_{2}, r\right) .
\end{aligned}
$$


The subindex $f$ indicates the pair density of the flat interface. The familiar Kirkwood-Buff expression for the surface tension of the planar interface ${ }^{2}$ is similarly found by comparing the zeroth-order terms in the expansion of $\sigma(R)$ in Eqs. (2.9) and (3.14),

$$
\sigma=\frac{1}{4} \int d z_{1} \int d \mathbf{r}_{12} u^{\prime}(r) r\left(1-3 s^{2}\right) \rho_{f}^{(2)}\left(z_{1}, z_{2}, r\right) \text {. }
$$

Equation (4.1) together with Eq. (4.2) gives a rigorous expression for the Tolman length. One may also derive an expression for $\delta$ using the expression for the pressure difference (2.14). Substituting $r=R+z_{1}$ into Eq. (2.14), and subsequently expanding to first order in the reciprocal radius, it can be seen that ${ }^{8}$

$$
\begin{aligned}
\Delta p=\frac{2}{R}\left[\int d z_{1}\left[p_{N}-p_{T}\right]_{f}\left(z_{1}\right)\right. \\
-\frac{1}{R}\left(\int d z_{1} z_{1}\left[p_{N}-p_{T}\right]_{f}\left(z_{1}\right)\right. \\
\left.\left.-\int d z_{1}\left[p_{N}-p_{T}\right]_{s, 1}\left(z_{1}\right)\right)\right] .
\end{aligned}
$$

Here the difference of the normal and tangential component of the pressure tensor has been expanded to first order in the reciprocal radius

$$
\begin{aligned}
{\left[p_{N}-p_{T}\right]\left(z_{1}\right)=} & {\left[p_{N}-p_{T}\right]_{f}\left(z_{1}\right) } \\
& +\frac{1}{R}\left[p_{N}-p_{T}\right]_{s, 1}\left(z_{1}\right) .
\end{aligned}
$$

From Eq. (4.3) we then get for the surface tension of the planar interface

$$
\sigma=\int d z_{1}\left[p_{N}-p_{T}\right]_{f}\left(z_{1}\right)
$$

and for Tolman's length

$$
\begin{aligned}
\delta= & \frac{1}{\sigma} \int d z_{1} z_{1}\left[p_{N}-p_{T}\right]_{f}\left(z_{1}\right) \\
& -\frac{1}{\sigma} \int d z_{1}\left[p_{N}-p_{T}\right]_{s, 1}\left(z_{1}\right) .
\end{aligned}
$$

The first term in this expression gives the position of the socalled mechanical surface of tension

$$
z_{s} \equiv \frac{1}{\sigma} \int d z_{1} z_{1}\left[p_{N}-p_{T}\right]_{f}\left(z_{1}\right) .
$$

In the literature ${ }^{2,3.5 .16}$ it is common to make no distinction between the mechanical surface of tension and the surface of tension defined by Tolman. This would imply that $\delta=-z_{s}$ so that the second contribution in Eq. (4.6) must be equal to $-2 z_{s}$. As we will show later this is not correct. Substituting the planar limit of $\left[p_{N}-p_{T}\right]\left(z_{1}\right)$ given in Eq. (3.9) into Eq. (4.7) gives

$$
\begin{aligned}
z_{s}= & \frac{1}{4 \sigma} \int d z_{1} \int d \mathbf{r}_{12} \int_{0}^{1} d \alpha u^{\prime}(r) r\left(1-3 s^{2}\right) z_{1} \\
& \times\left\{\rho_{f}^{(2)}\left(z_{1}-\alpha s r, z_{1}+(1-\alpha) s r, r\right)-\rho_{f ; 1, g}^{(2)}\left(z_{1}, r\right)\right\} .
\end{aligned}
$$

As was discussed in Sec. III it is important to subtract the extrapolated pair density from the pair density in the interfa- cial region in this equation, before shifting the integration over $z_{1}$ over a distance $\alpha s r$. Substituting $z_{1}^{\prime}=z_{1}+\alpha s r$ and subsequently dropping the prime one finds

$$
\begin{aligned}
z_{s}= & \frac{1}{4 \sigma} \int d z_{1} \int d \mathbf{r}_{12} \int_{0}^{1} d \alpha u^{\prime}(r) r\left(1-3 s^{2}\right) \\
& \times\left(z_{1}+\alpha s r\right) \rho_{f}^{(2)}\left(z_{1}, z_{2}, r\right) \\
& +\frac{1}{4 \sigma} \int d z_{1} \int d \mathbf{r}_{12} \int_{0}^{1} d \alpha u^{\prime}(r) r\left(1-3 s^{2}\right) \\
& \times\left(z_{1}+\alpha s r\right)\left[\rho_{f: l, g}^{(2)}\left(z_{1}, r\right)-\rho_{f:,, g}^{(2)}\left(z_{1}+\alpha s r, r\right)\right] .
\end{aligned}
$$

In the first term we can carry out the integration over $\alpha$, while in the second term we subsequently integrate over $z_{1}$, $\alpha$, and $s$. We find

$$
\begin{aligned}
z_{s}= & \frac{1}{8 \sigma} \int d z_{1} \int d \mathbf{r}_{12} u^{\prime}(r) r\left(1-3 s^{2}\right) \\
& \times\left(z_{1}+z_{2}\right) \rho_{f}^{(2)}\left(z_{1}, z_{2}, r\right) \\
& +\frac{2 \pi}{45 \sigma} \int d r u^{\prime}(r) r^{5}\left[\rho_{f: s}^{(2)}(r)-\rho_{f: l}^{(2)}(r)\right]
\end{aligned}
$$

With the use of Eq. (4.1) we see that $z_{\mathrm{s}}=-\delta+A$ where

$$
A \equiv \frac{2 \pi}{45 \sigma} \int d r u^{\prime}(r) r^{5}\left[\rho_{f: g}^{(2)}(r)-\rho_{f: l}^{(2)}(r)\right]
$$

As $A$ is, in general, unequal to zero, unlike a term of a similar origin which appeared in the expression for the pressure difference [cf. Eq. (3.12)], this result shows that Tolman's length does not give the location of the mechanical surface of tension.

The molecular dynamics results from Nijmeijer et al. ${ }^{8}$ for Tolman's length and the location of the mechanical surface of tension at one particular temperature (with a scaled value of 0.90 ) are

$$
\delta=-0.3 \pm 0.9, \quad z_{s}=-2.5 \pm 0.1 .
$$

These lengths are scaled with the Lennard-Jones radius. Their value for $\delta$ is obtained from a direct calculation of $\Delta p$ for a number of droplet radii as well as from a microscopic calculation of the integral in Eq. (4.6) for a large number of configurations. Their value for $z_{s}\left(I_{1}^{\infty}\right.$ in their notation $)$ is found by a microscopic calculation of the integral in Eq. (4.7) for a large number of configurations. Within the error they clearly find that the distance between the mechanical surface of tension and the Gibbs surface is not given by Tolman's length.

Using a simple model for the pair density that was also used in a previous paper to calculate the spontaneous curvature and the rigidity constants, we now calculate $\delta$ and $A$ from Eqs. (4.1) and (4.11). The model assumes the pair density to be of the form

$$
\rho_{f}^{(2)}\left(z_{1}, z_{2}, r\right)=\rho\left(z_{1}\right) \rho\left(z_{2}\right) g(r),
$$

where $g(r)$ is the pair correlation function in the uniform liquid. For the density profile we consider the classical van der Waals profile

$$
\rho(z)=\rho_{c}-\frac{\Delta \rho}{2} \tanh (z / 2 \xi)
$$


where $\rho_{c} \equiv \frac{1}{2}\left(\rho_{l}+\rho_{g}\right), \Delta \rho \equiv\left(\rho_{l}-\rho_{g}\right)$, and $\xi$ is the bulk correlation length, which is a measure of the thickness of the interface. We note that Eq. (4.14) fulfills the requirement that the excess number density is zero. Using Eqs. (4.13) and (4.14) into the expression for the Tolman length we show in Appendix B that, on the basis of the symmetry of the model pair density, $\delta=0$. This is in agreement with the value of Nijmeijer et al. $(\delta=-0.3 \pm 0.9)$. For $A$ one finds

$$
A=\frac{-4 \pi}{45 \sigma} \rho_{c} \Delta \rho \int d r u^{\prime}(r) r^{5} g(r) \text {. }
$$

Substituting the same model for the pair density into Eq. (4.2) for the surface tension and assuming that $\xi$ is large compared to the interaction range, an assumption most appropriate close to the critical point, the surface tension is shown to be given by

$$
\sigma=\frac{\Delta \rho^{2}}{\xi} \frac{\pi}{45} \int d r u^{\prime}(r) r^{5} g(r)
$$

in Ref. 14. Combining Eqs. (4.15) and (4.16) it follows that close to the critical point, $A$ is given by

$$
A=-4 \rho_{c} \xi / \Delta \rho \text {. }
$$

From this equation one directly obtains the scaling behavior as a function of the reduced temperature of $A$ close to the critical point. As $\Delta \rho \sim t^{\beta}$ and $\xi \sim t^{-\nu}$ it follows that $A \sim t^{-\beta-v}$. For the temperature considered in the MD calculations of Nijmeijer $e t$ al. it seems not unreasonable to obtain an approximate value for $A$ using Eq. (4.17). From Nijmeijer et al. we find for $\Delta \rho=0.62 \pm 0.005$ and $\rho_{c}=0.36 \pm 0.005$ in reduced units. The thickness $\xi=0.90 \pm 0.05$ can be obtained by fitting their density profiles with the tanh profile. The resulting value for the location of the mechanical surface of tension then becomes, $\delta=0$,

$$
z_{s}=A=-2.1 \pm 0.1
$$

In view of the assumptions made in the calculation of this number, the agreement with the value from molecular dynamics $\left(z_{s}=-2.5 \pm 0.1\right)$ is rather satisfactory.

\section{SUMMARY}

The main results found in this paper are listed as follows.

The use of the Irving-Kirkwood expression for the pressure tensor leads to an expression for the pressure difference which agrees to third order in the reciprocal equimolar radius with the expression found using microscopic sum rules. In particular, the expressions for the surface tension of the flat interface and the Tolman length are found to agree.

The use of the Schofield-Henderson expression for the pressure tensor for a particular contour different from the contour that leads to the Irving-Kirkwood expression, gives incorrect results for the pressure difference and in particular for Tolman's length. Clearly, the possible validity of the Schofield-Henderson expression for the pressure tensor depends on the choice of the contour.

The distance between the mechanical surface of tension and the Gibbs dividing surface is not given by Tolman's length. The mechanical surface of tension does therefore not coincide with the surface of tension defined by Tolman.

Using an approximate expression for the pair density it is possible to find values for the location of the surface of tension and for Tolman's length which are in reasonably good agreement with numerical values obtained by $\mathrm{Nij}$ meijer et $a l^{8}$

As a last remark we want to emphasize that, even though this paper may be considered as to support the soundness of the Irving-Kirkwood expression for the pressure tensor in an inhomogeneous system, the nonunique nature of this quantity is still a matter of concern. Thus we consider it advisable not to use the pressure tensor whenever this can be avoided.

\section{ACKNOWLEDGMENTS}

We would like to thank Professor H. N. W. Lekkerkerker for interesting discussions and for stressing the importance to us of verifying to what extent our earlier results can be reproduced by following the pressure tensorial route. This work is part of the research program of the Leiden Material Science Center and of the "Stichting voor Fundamenteel Onderzoek der Materie" (FOM).

\section{APPENDIX A: CALCULATION OF $\triangle p$ TO FIRST ORDER IN 1/R USING A DIFFERENT PATH IN EQ. (2.16)}

The path we use to calculate the normal and tangential components of the pressure tensor in Eq. (2.16) is given by a line from $r_{1}=\left(r_{1, \|}, z_{1}\right)$ to $\left(r_{2, \|}, z_{1}\right)$ and then from $\left(r_{2, \|}, z_{1}\right)$ to $\mathbf{r}_{2}=\left(\mathbf{r}_{2, \|}, z_{2}\right)$,

$\mathbf{I}=\left(\alpha \mathbf{r}_{12, \|}, 0\right)$ followed by $\mathbf{I}=\left(\mathbf{r}_{12, \|}, \alpha s r\right)$ with $0<\alpha<1$.

The normal and tangential component of the pressure tensor are then given by

$$
\begin{aligned}
& p_{N}(z)=k_{B} T \rho(z)-\frac{1}{2} \int d \mathbf{r}_{12} \int_{0}^{1} d \alpha u^{\prime}(r) r s^{2} \rho_{s}^{(2)}\left(\left|(z-\alpha s r) \hat{z}-\mathbf{r}_{12, \|}\right|,|[z+(1-\alpha) s r] \hat{z}|, r\right), \\
& p_{r}(z)=k_{B} T \rho(z)-\frac{1}{4} \int d \mathbf{r}_{12} \int_{0}^{1} d \alpha u^{\prime}(r) r\left(1-s^{2}\right) \rho_{s}^{(2)}\left(\left|z \hat{z}-\alpha \mathbf{r}_{12, \|}\right|,\left|(z+s r) \hat{z}+(1-\alpha) \mathbf{r}_{12, \|}\right|, r\right),
\end{aligned}
$$

where again the $z$ axis has been chosen in the direction of $\mathbf{r}$ so that $\mathbf{r}=z \hat{z}$. The arguments of the pair density in the above expressions are, to first order in $r / R$, given by 


$$
\begin{aligned}
& \left|(z-\alpha s r) \hat{z}-\mathbf{r}_{12, \|}\right|=z-\alpha s r+\frac{r^{2}}{2 z}\left(1-s^{2}\right), \\
& |[z+(1-\alpha) s r] \hat{z}|=z+(1-\alpha) s r \\
& \left|z \hat{z}-\alpha \mathbf{r}_{12, \|}\right|=z+\frac{r^{2}}{2 z} \alpha^{2}\left(1-s^{2}\right) \\
& \left|(z+s r) \hat{z}+(1-\alpha) \mathbf{r}_{12, \|}\right|=z+s r+\frac{r^{2}}{2 z}(1-\alpha)^{2}\left(1-s^{2}\right)
\end{aligned}
$$

We expand the pair densities around the pair densities with the first and second argument replaced by the first two terms in Eq. (A3),

$$
\begin{aligned}
& \rho_{s}^{(2)}\left(\left|(z-\alpha s r) \hat{z}-\mathbf{r}_{12, \| \mid}\right|,|[z+(1-\alpha) s r] \hat{z}|, r\right) \\
& \quad=\rho_{s}^{(2)}(z-\alpha s r, z+(1-\alpha) s r, r)+\rho_{1}^{(2)}(z-\alpha s r, z+(1-\alpha) s r, r) \frac{r^{2}}{2 z}\left(1-s^{2}\right), \\
& \rho_{s}^{(2)}\left(\left|z \hat{z}-\alpha \mathbf{r}_{12, \|}\right|,\left|(z+s r) \hat{z}+(1-\alpha) \mathbf{r}_{12, \| \mid}\right|, r\right) \\
& \quad=\rho_{s}^{(2)}(z, z+s r, r)+\rho_{1}^{(2)}(z, z+s r, r) \frac{r^{2}}{2 z} \alpha^{2}\left(1-s^{2}\right)+\rho_{2}^{(2)}(z, z+s r, r) \frac{r^{2}}{2 z}(1-\alpha)^{2}\left(1-s^{2}\right) .
\end{aligned}
$$

Here, again, higher-order derivatives in the above expansion correspond to higher-order powers in $r / R$ and the subindices of the pair density indicate partial differentiation with respect to its first or second argument. The partial differentiation with respect to the first and second argument are written in terms of a differentiation with respect to $s$ and $z$,

$$
\begin{aligned}
& \rho_{1}^{(2)}(z-\alpha s r, z+(1-\alpha) s r, r)=\frac{-1}{r} \frac{d}{d s} \rho_{s}^{(2)}(z-\alpha s r, z+(1-\alpha) s r, r)+(1-\alpha) \frac{d}{d z} \rho_{s}^{(2)}(z-\alpha s r, z+(1-\alpha) s r, r), \\
& \rho_{1}^{(2)}(z, z+s r, r)=-\frac{1}{r} \frac{d}{d s} \rho_{s}^{(2)}(z, z+s r, r)+\frac{d}{d z} \rho_{s}^{(2)}(z, z+s r, r) \\
& \rho_{2}^{(2)}(z, z+s r, r)=\frac{1}{r} \frac{d}{d s} \rho_{s}^{(2)}(z, z+s r, r)
\end{aligned}
$$

We can now insert Eqs. (A4) and (A5) into the expressions for the normal and tangential component of the pressure tensor of Eq. (A2). The resulting expressions are inserted into the expression for $\Delta p$ in Eq. (2.14). Writing again $z \equiv R+z_{1}$ we find

$$
\begin{aligned}
\Delta p_{H}= & \frac{2}{R} \int d z_{1}\left(1-\frac{z_{1}}{R}\right)\left[p_{N}\left(z_{1}\right)-p_{T}\left(z_{1}\right)\right]=\frac{1}{2 R} \int d \mathbf{r}_{12} \int d z_{1} \int_{0}^{1} d \alpha u^{\prime}(r) r \\
& \times\left[-2 s^{2}\left(1-\frac{z_{1}}{R}-\frac{r}{2 R}\left(1-s^{2}\right) \frac{d}{d s}+\frac{r^{2}}{2 R}\left(1-s^{2}\right)(1-\alpha) \frac{d}{d z_{1}}\right) \rho_{s}^{(2)}\left(z_{1}-\alpha s r, z_{1}+(1-\alpha) s r, r\right)\right. \\
& \left.+\left(1-s^{2}\right)\left(1-\frac{z_{1}}{R}+\frac{r}{2 R}(1-2 \alpha)\left(1-s^{2}\right) \frac{d}{d s}+\frac{r^{2}}{2 R} \alpha^{2}\left(1-s^{2}\right) \frac{d}{d z_{1}}\right) \rho_{s}^{(2)}\left(z_{1}, z_{1}+s r, r\right)\right] .
\end{aligned}
$$

The subindex $H$ of the pressure difference indicates that this pressure difference is derived using the path in Eq. (A1) which leads to the Harasima expression for the pressure tensor of the flat interface. We can now perform the partial integration over $s$ and $z_{1}$ to yield

$$
\begin{aligned}
\Delta p_{H}= & \frac{1}{2 R} \int d \mathbf{r}_{12} \int d z_{1} \int_{0}^{1} d \alpha u^{\prime}(r) r\left[\left(-2 s^{2}+2 s^{2} \frac{z_{1}}{R}-\frac{2 r s}{R}\left(1-2 s^{2}\right)\right) \rho_{s}^{(2)}\left(z_{1}-\alpha s r, z_{1}+(1-\alpha) s r, r\right)\right. \\
& \left.+\left(1-s^{2}\right)\left(1-\frac{z_{1}}{R}+\frac{2 r s}{R}(1-2 \alpha)\right) \rho_{s}^{(2)}\left(z_{1}, z_{1}+s r, r\right)\right] \\
& +\frac{1}{2 R} \int d \mathbf{r}_{12} \int_{0}^{1} d \alpha u^{\prime}(r) r\left[-\frac{r^{2} s^{2}}{R}\left(1-s^{2}\right)(1-\alpha) \rho_{s}^{(2)}\left(z_{1}-\alpha s r, z_{1}+(1-\alpha) s r, r\right)\right. \\
& \left.+\frac{r^{2}}{2 R} \alpha^{2}\left(1-s^{2}\right)^{2} \rho_{s}^{(2)}\left(z_{1}, z_{1}+s r, r\right)\right] \begin{array}{l}
z_{1}=\infty \\
z_{1}=-\infty
\end{array}
\end{aligned}
$$

We shift the integration over $z_{1}$ over a distance $\alpha s r$, i.e., $z_{1} \rightarrow z_{1}+\alpha s r$, in the first part of the first term of the above expression and simplify the second term. We then derive 


$$
\begin{aligned}
\Delta p_{H}= & \frac{1}{2 R} \int d \mathbf{r}_{12} \int d z_{1} \int_{0}^{1} d \alpha u^{\prime}(r) r\left[\left(1-3 s^{2}\right)\left(1-\frac{z_{1}}{R}\right)+\frac{2 r s^{3}}{R}-\frac{2 \alpha r s}{R}\left(2-3 s^{2}\right)\right] \rho_{s}^{(2)}\left(z_{1}, z_{1}+s r, r\right) \\
& +\frac{1}{2 R} \int d \mathbf{r}_{12} \int d z_{1} \int_{0}^{1} d \alpha u^{\prime}(r) r\left[-2 s^{2}+\frac{2 s^{2}}{R}\left(z_{1}+\alpha s r\right)-\frac{2 r s}{R}\left(1-2 s^{2}\right)\right]\left[\rho_{s, l, g}^{(2)}\left(z_{1}, r\right)-\rho_{s, l, g}^{(2)}\left(z_{1}+\alpha s r, r\right)\right] \\
& +\frac{1}{2 R^{2}} \int d \mathbf{r}_{12} \int_{0}^{1} d \alpha u^{\prime}(r) r^{3}\left(1-s^{2}\right)\left[-(1-\alpha) s^{2}+\frac{\alpha^{2}}{2}\left(1-s^{2}\right)\right]\left[\rho_{s, g}^{(2)}(r)-\rho_{s, l}^{(2)}(r)\right] .
\end{aligned}
$$

In the first term the integration over $\alpha$ can be carried out while in the second term we subsequently integrate over $\alpha$ and $s$. In the last term we integrate over $\alpha$ and $s$ and combine the result with the result of the second term. We are then finally left with

$$
\begin{aligned}
\Delta p_{H}= & \frac{1}{2 R} \int d \mathbf{r}_{12} \int d z_{1} u^{\prime}(r) r\left[\left(1-3 s^{2}\right)\left(1-\frac{z_{1}}{R}\right)\right. \\
& \left.-\frac{r s}{R}\left(2-5 s^{2}\right)\right] \rho_{s}^{(2)}\left(z_{1}, z_{1}+s r, r\right)-\frac{2 \pi}{9 R^{2}} \int d r u^{\prime}(r) r^{5}\left[\rho_{s, g}^{(2)}(r)-\rho_{s, l}^{(2)}(r)\right] .
\end{aligned}
$$

In order for us to compare this result with the previous derived result for the pressure difference using the Irving-Kirkwood expression for the pressure tensor we use the identity

$$
\int d \mathbf{r}_{12} \int d z_{1} f(s)\left[\rho_{s}^{(2)}\left(z_{1}, z_{1}+s r, r\right)-\rho_{s, l, g}^{(2)}\left(z_{1}, r\right)\right]=\frac{1}{2} \int d \mathbf{r}_{12} s r f(s)\left[\rho_{s ; g}^{(2)}(r)-\rho_{s, l}^{(2)}(r)\right],
$$

where we require that $f(s)=-f(-s)$. This result can be derived using the fact that the pair density is symmetric under interchanging the first two arguments. Equation (A9) can then be written as

$$
\Delta p_{H}=\frac{1}{2 R} \int d \mathbf{r}_{12} \int d z_{1} u^{\prime}(r) r\left(1-3 s^{2}\right)\left(1-\frac{z_{1}}{R}\right) \rho_{s}^{(2)}\left(z_{1}, z_{1}+s r, r\right)+\frac{\pi}{9 R^{2}} \int d r u^{\prime}(r) r^{5}\left(\rho_{s ; g}^{(2)}(r)-\rho_{s ; l}^{(2)}(r)\right)
$$

This expression should be compared with the exact result for the pressure difference in Eq. (3.13) to first order in the reciprocal radius. We use Eq. (A10) to make the difference between the two expressions more transparent. The IrvingKirkwood result for the pressure difference is then

$$
\Delta p=\frac{1}{2 R} \int d \mathbf{r}_{12} \int d z_{1} u^{\prime}(r) r\left(1-3 s^{2}\right)\left(1-\frac{z_{1}}{R}\right) \rho_{s}^{(2)}\left(z_{1}, z_{1}+s r, r\right)-\frac{2 \pi}{15 R^{2}} \int d r u^{\prime}(r) r^{5}\left[\rho_{s ; 3}^{(2)}(r)-\rho_{s, l}^{(2)}(r)\right]
$$

Although the first terms in Eqs. (A11) and (A12) are equal and thus lead to the same expression for the surface tension of the flat interface, the last terms are clearly not equal. As is clear from the discussion in Sec. IV, the difference between the two expressions is proportional to $A$ and therefore unequal to zero.

\section{APPENDIX B: CALCULATION OF TOLMAN'S LENGTH USING THE SIMPLE APPROXIMATION FOR THE PAIR DENSITY}

We will now show that $\delta$ becomes equal to zero when we insert the approximate expression for the pair density of Eq. (4.13) together with Eq. (4.14), into the formula in Eq. (4.1). Subtracting the extrapolated pair density in this formula gives us

$\delta=\frac{-1}{8 \sigma} \int d z_{1} \int d \mathbf{r}_{12} u^{\prime}(r) r\left(1-3 s^{2}\right)\left(2 z_{1}+s r\right)\left[\rho_{f}^{(2)}\left(z_{1}, z_{2}, r\right)-\rho_{f: l, g}^{(2)}\left(z_{1}, r\right)\right]$.

The term in square brackets, the excess pair density, can be written as the sum of four contributions

$$
\begin{aligned}
& \rho_{f}^{(2)}\left(z_{1}, z_{2}, r\right)-\rho_{f: l, g}^{(2)}\left(z_{1}, r\right) \\
&=\left(\rho_{c}^{2}-\frac{1}{2} \rho_{c} \Delta \rho\left[\tanh \left(z_{1} / 2 \xi\right)+\tanh \left(z_{2} / 2 \xi\right)\right]\right. \\
&\left.+\frac{\Delta \rho^{2}}{4} \tanh \left(z_{1} / 2 \xi\right) \tanh \left(z_{2} / 2 \xi\right)\right) g(r)-\left(\rho_{c}^{2}-\rho_{c} \Delta \rho \operatorname{sgn}\left(z_{1}\right)+\frac{\Delta \rho^{2}}{4}\right) g(r) \\
&=-\frac{1}{2} \rho_{c} \Delta \rho\left[\tanh \left(z_{1} / 2 \xi\right)-\operatorname{sgn}\left(z_{1}\right)\right] g(r)-\frac{1}{2} \rho_{c} \Delta \rho\left[\tanh \left(z_{2} / 2 \xi\right)-\operatorname{sgn}\left(z_{2}\right)\right] g(r) \\
&-\frac{1}{2} \rho_{c} \Delta \rho\left[\operatorname{sgn}\left(z_{2}\right)-\operatorname{sgn}\left(z_{1}\right)\right] g(r)+\frac{\Delta \rho^{2}}{4}\left[\tanh \left(z_{1} / 2 \xi\right) \tanh \left(z_{2} / 2 \xi\right)-1\right] g(r),
\end{aligned}
$$

where sgn denotes the sign function which equals the sign of the argument. Inserting this equation in Eq. (B1) one finds 


$$
\begin{aligned}
\delta= & \frac{1}{16 \sigma} \rho_{c} \Delta \rho \int d z_{1} \int d \mathbf{r}_{12} u^{\prime}(r) r g(r)\left(1-3 s^{2}\right)\left(2 z_{1}+s r\right)\left[\tanh \left(z_{1} / 2 \xi\right)-\operatorname{sgn}\left(z_{1}\right)\right] \\
& +\frac{1}{16 \sigma} \rho_{c} \Delta \rho \int d z_{1} \int d \mathbf{r}_{12} u^{\prime}(r) r g(r)\left(1-3 s^{2}\right)\left(2 z_{1}+s r\right)\left\{\tanh \left[\left(z_{1}+s r\right) / 2 \xi\right]-\operatorname{sgn}\left(z_{1}+s r\right)\right\} \\
& +\frac{1}{16 \sigma} \rho_{c} \Delta \rho \int d z_{1} \int d \mathbf{r}_{12} u^{\prime}(r) r g(r)\left(1-3 s^{2}\right)\left(2 z_{1}+s r\right)\left[\operatorname{sgn}\left(z_{1}+s r\right)-\operatorname{sgn}\left(z_{1}\right)\right] \\
& -\frac{\Delta \rho^{2}}{32 \sigma} \int d z_{1} \int d \mathbf{r}_{12} u^{\prime}(r) r g(r)\left(1-3 s^{2}\right)\left(2 z_{1}+s r\right)\left\{\tanh \left(z_{1} / 2 \xi\right) \tanh \left[\left(z_{1}+s r\right) / 2 \xi\right]-1\right\} .
\end{aligned}
$$

In the first term the integration over $s$ can be carried out to yield zero. In the second term the integration can also be carried out after shifting $z_{1}$, i.e., $z_{1} \rightarrow z_{1}-s r$. The result, again, is zero. The last term vanishes because of the symmetry of the profile; changing $z_{1} \rightarrow-z_{1}$ and $s \rightarrow-s$ and using $\tanh (x)=-\tanh (-x)$ we find that the integrand is antisymmetric under this operation. This term thus vanishes due to the symmetry of the density profile, if the density profile would not have the property of being antisymmetric, which is generally not the case, Tolman's length is not necessarily zero. The fact that the symmetry of the density profile leads to a vanishing Tolman length has also been found in density-functional calculations of $\delta$ by Fisher and Wortis. ${ }^{18}$ The integration over $z_{1}$ can be carried out in the third term in Eq. (B3),

$$
\delta=\frac{-1}{8 \sigma} \rho_{c} \Delta \rho \int d \mathbf{r}_{12} u^{\prime}(r) r g(r)\left(1-3 s^{2}\right)\left(\int_{0}^{-s r} d z_{1} 2 z_{1}+s r \int_{0}^{-s r} d z_{1}\right)=0 .
$$

In this derivation we see that the sum of the two integrals in large parentheses in Eq. (B4) is equal to zero but that both integrals itself are not. Previous derived equations for Tolman's length ${ }^{2}$ have in the past led to nonzero values for $\delta$ using exactly the same model for the pair density due to the absence of the second term ( $s r)$ in the expression for $\delta$ in Eq. (B1).

${ }^{1}$ J. W. Gibbs, Collected Works (Dover, New York, 1961), Vol. 2.

${ }^{2}$ J. G. Kirkwood and F. P. Buff, J. Chem. Phys. 17, 338 (1949).

${ }^{3}$ F. P. Buff, J. Chem. Phys. 23, 419 (1955).

${ }^{4}$ W. Helfrich, Z. Naturforsch. 28c, 693 (1973).

${ }^{5}$ See, e.g., J. S. Rowlinson and B. Widom, Molecular Theory of Capillarity (Clarendon, Oxford 1982).

${ }^{\circ}$ R. C. Tolman, J. Chem. Phys. 17, 333 (1949).

${ }^{7}$ S. M. Thompson, K. E. Gubbins, J. P. R. B. Walton, R. A. R. Chanty, and J. S. Rowlinson, J. Chem. Phys. 81, 530 (1984).

${ }^{8}$ M. J. P. Nijmeijer, C. Bruin, A. B. Woerkom, A. F. Bakker, and J. M. J. van Leeuwen, J. Chem. Phys. 96, 565 (1992).
${ }^{9}$ J. H. Irving and J. G. Kirkwood, J. Chem. Phys. 18, 817 (1950).

${ }^{10}$ P. Schofield and J. R. Henderson, Proc. R. Soc. London Ser. A. 379, 231 (1982).

"M. Baus and R. Lovett, Phys. Rev. A 44, 1211 (1991).

${ }^{12}$ J. S. Rowlinson, Phys. Rev. Lett. 67, 406 (1991).

${ }^{13}$ M. Baus and R. Lovett, Phys. Rev. Lett. 67, 407 (1991).

${ }^{14}$ E. M. Blokhuis and D. Bedeaux, Physica A 184, 42 (1992).

${ }^{15}$ A. Harasima, Adv. Chem. Phys. 1, 203 (1958).

${ }^{16}$ S. Ono and S. Kondo, Handb. Phys. 10, 134 (1960).

${ }^{17} \mathrm{M}$. Baus (private communications).

${ }^{18}$ M. P. A. Fischer and M. Wortis, Phys. Rev. B 29, 6252 (1984). 